\title{
Effect of Interval Time between Corrosive and Abrasive Challenges on a Nanoparticulate Composite Resin
}

\author{
Ana Laura Rezende Vilela ${ }^{1}$ Alexandre Coelho Machado ${ }^{2}$ Lucas Lemes Queiroz ${ }^{1}$ \\ Pedro Henrique Mauro Batista ${ }^{1}$ André Luís Faria-e-Silva ${ }^{3}$ Murilo de Sousa Menezes ${ }^{1}$ \\ ${ }^{1}$ Department of Restorative Dentistry and Dental Materials, Dental \\ Address for correspondence Murilo de Sousa Menezes, DDS, MSc, \\ School, Federal University of Uberlândia, Brazil \\ ${ }^{2}$ Technical Health School, Federal University of Uberlândia, Brazil \\ $\mathrm{PhD}$, Department of Restorative Dentistry and Dental Materials, \\ ${ }^{3}$ Department of Restorative Dentistry, Dental School, Federal \\ Dental School, Federal University of Uberlandia, Avenida Pará, 1720, \\ Bloco 4L, Anexo A, Sala 34, Campus Umuarama Uberlândia - Minas \\ University of Sergipe, Brazil \\ Gerais-Brazil (e-mail: murilomenezes@ufu.br).
}

Eur J Dent 2021;15:607-611.

\begin{abstract}
Keywords

- composite resins

- toothbrushing

- beverages
\end{abstract}

Objective This study aimed to evaluate the effect of interval time after acidic beverage intake and brushing on roughness and hardness of resin composite.

Materials and Methods Nanofilled resin composites were tested as per interval time (no interval, 15 or 30 minutes) between aging media (isotonic, sports drink) and brushing. Specimens $(n=9)$ were subjected to three cycles daily for 5 days with immersion in beverage followed by simulated brushing ( 585 strokes). The brushing (control) group was submitted only in brushing cycles. Roughness and microhardness were analyzed in the baseline and end of the experiment. Surface morphology was analyzed by using scanning electron microscopy (SEM).

Statistical analysis Data were analyzed by one-way ANOVA and Tukey's honestly significant difference $(\alpha=0.05)$.

Results Roughness was higher in no interval group and lower in 30 minutes and control. The 15 minutes present no statistical difference between control, 30 minutes and no interval. The hardness not present difference between groups. The SEM showed the no interval more roughness than 15 and 30 minutes, control and baseline.

Conclusion The interval time between erosive and abrasive challenge is important to preserve the smoothness surface of composite resin.

\section{Introduction}

The composite resin restorations are widely indicated for the treatment of caries, ${ }^{1}$ esthetic reasons, and dental wear management. ${ }^{1}$ The frequent use of composite resins are related to greater preservation of the dental structure, low cost, simple technique, and lower clinical time. ${ }^{2}$ This material consists of an organic matrix, filler inorganic particles, and silane bonding agent. ${ }^{3}$ The size, shape, and amount of the filler particles improve mechanic properties and polishing. ${ }^{2}$ Currently, the smallest scale of particles used are the nanofillers and the composite resins with exclusivity of nanometric particles are classified as nanofilled. Moreover, the annual failure rates of composite resin restorations reaches 4.1 and $2.2 \%$ for anterior and posterior teeth. ${ }^{3}$ published online

September 7, 2021
DoI https://doi.org/ $10.1055 / \mathrm{s}-0041-1726161$ ISSN 1305-7456.
(C) 2021. European Journal of Dentistry.

This is an open access article published by Thieme under the terms of the Creative Commons Attribution-NonDerivative-NonCommercial-License, permitting copying and reproduction so long as the original work is given appropriate credit. Contents may not be used for commercial purposes, or adapted, remixed, transformed or built upon. (https://creativecommons.org/licenses/by-nc-nd/4.0/)

Thieme Medical and Scientific Publishers Pvt. Ltd., A-12, 2nd Floor, Sector 2, Noida-201301 UP, India 
The wide range of clinical applications of composite resin is assessed mainly through their ability to mimic the optical effects of dental structures, mechanical, adhesion to dental structures, and physical properties. ${ }^{2}$ Despite all these advantages, the resin composite restorations are susceptible to surface changes due to aging caused by acidic abrasive substances. These aging can affect the aesthetic properties and also the smoothness and strength of the restoration, becoming unsatisfactory over the time. According to World Dental Federation (FDI), the failures criteria for direct restoration are surface luster, staining, color match, translucency, and esthetic anatomical form. ${ }^{4}$ The main causes for these undesirable effects can be assigned to insufficient polymerization, unsatisfactory finishing and polishing, parafunctional habits, operator experience, and incorrect and unsatisfactory oral hygiene. $^{5}$

The roughness or smoothness surface are related to polishing technique. A deficiency in the finishing, inadequate polishing procedure, acidic diet, and deleterious brushing habits increases the roughness surface over the time, resulting in aesthetic damage to the restoration. ${ }^{2}$ Moreover, a rougher surface increase the potential biofilm accumulation, ${ }^{6}$ increasing the gingival inflammation and maintaining color stability for less time. ${ }^{2}$ The oral hygiene is important to teeth and periodontal healthy, through of removing dental plaque and superficial staining on teeth and composite resin restoration. ${ }^{5}$ The abrasives present in toothpaste have function of contribute to biofilm remove and reduce restorations and teeth pigmentation after eating food and drinks. ${ }^{7}$ However, toothbrush and toothpaste can negatively affect the smoothness surface of composite resin according to the abrasiveness of the dentifrice, stiffness of toothbrush bristles, and major associated with acidic diet. ${ }^{8}$

The consumption of sports and energy drinks is currently increasing, mainly due to the population's greater concern about health and body aesthetics. ${ }^{9}$ Moreover, beverages present low $\mathrm{pH}(3.8-2.3)$ generates dental corrosion ${ }^{10}$ and cause degradation of the composite resin organic matrix, increasing the surface roughness and decreasing hardness and flexural strength. ${ }^{11}$ The consumption of acidic beverages and oral hygiene is a daily practice of the majority of the world population. The other factor that may be associated with the wear of composite resins is through brushing with high abrasive dentifrice, as whitening toothpastes. ${ }^{12}$

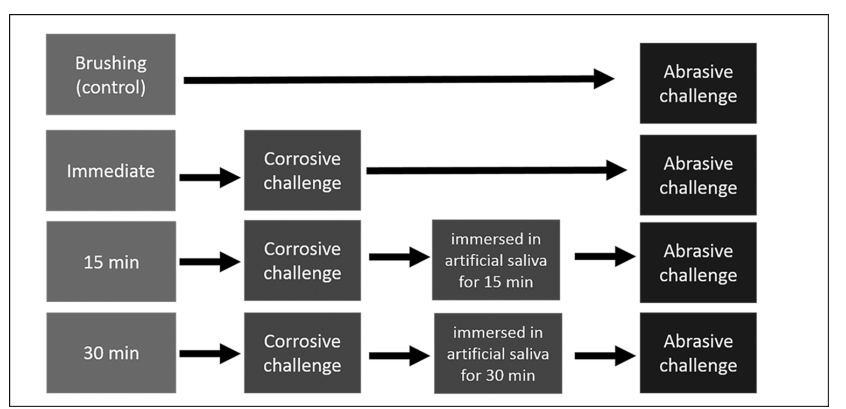

Fig. 1 Experimental designs defined according to time elapsed between corrosive and/or abrasive challenges.
Therefore, the objective of this article is to evaluate the influence of interval time between corrosive and abrasive challenge on surface roughness and hardness of nanofilled composite resins. The null hypothesis of the study is the interval time between acidic beverage and brushing will not affect the surface roughness and hardness of composite resins.

\section{Materials and Methods}

\section{Experimental Design}

This was a laboratory study conducted to evaluate the independent variable "time elapsed between corrosive and abrasive challenges" on changes in roughness and hardness (dependent variables). Four levels of independent variable were defined based on interval time between the challenges (no interval, 15 or 30 minutes) and absence of corrosive challenge (control; - Fig. 1).

\section{Specimen Preparation}

Thirty-six disc-shaped specimens of the nanofilled composite resin Filtek Z350 X (3M ESPE, St. Paul, Minnesota, United States), shade A1, were built up by using a cylindrical teflon mold ( $6 \mathrm{~mm}$ of diameter and $1 \mathrm{~mm}$ of thickness). After the composite insertion, the mold was covered with mylar strips, and the material maintained under digital pressure for 10 seconds before the light activation. The specimens were light cured for 20 seconds with the light-emitting diode Bluephase N (Ivoclar Vivadent, Schaan, Liechtenstein; irradiance $=1,000 \mathrm{~mW} / \mathrm{cm}^{2}$ ). The polymerized specimens were stored in distilled water at $37^{\circ} \mathrm{C}$ for 24 hours before the finishing procedures with $\mathrm{SiC}$ sandpapers $(600,800,1,200$, and 2,000) under water irrigation (Politriz Universal, Arotec, São Paulo, SP, Brazil). Following, the specimens were cleaned in an ultrasonic bath with distilled water for 10 minutes and stored in artificial saliva at $37^{\circ} \mathrm{C}$.

\section{Measurements at Baseline}

The average surface roughness $(\mathrm{Ra})$ of the specimens was assessed by using a surface roughness tester (Surftest SJ- 410; Mitutoyo Corp, Tokyo, Japan) at a constant speed of $0.25 \mathrm{~mm} / \mathrm{s}$ and cut-off of $0.8 \mathrm{~mm}$. Three readings were carried out for each specimen modifying direction at approximately 120 degrees between two consecutive readings. The mean surface roughness of the three readings was recorded for each specimen. Moreover, the knoop microhardness of specimens was determined by using a microhardness tester (FM-7000, Future-Tech Corp, Kawasaki, Japan) with a diamond Knoop indenter. Five equidistant indentations were carried out with a load of $0.98 \mathrm{~N}$ for 15 seconds on the specimen's surface, and averaged hardness was recorded for each specimen.

\section{Corrosive Challenge}

Twenty-seven specimens were immersed for 5 minutes in $5 \mathrm{~mL}$ of lemon flavor isotonic beverages (PowerADE Lemon, Coca-Cola, Atlanta, Georgia, United States) with agitation of $120 \mathrm{rpm},{ }^{11}$ three times a day for 5 days. The $\mathrm{pH}$ of beverages 
( 3.8) was measured daily. The nine remaining specimens were used as a control. Following, the specimens underwent to the corrosive challenge were kept in artificial saliva for either 15 or 30 minutes before the abrasive challenge, or abraded under toothbrushing movements without any interval $(n=9)$.

\section{Abrasive Challenge}

All specimens were brushed by using soft toothbrushes (Colgate Classic, Colgate-Palmolive, São Bernardo do Campo, SP, Brazil) attached to a toothbrushing simulator machine (Odeme Biotechnology, Joaçaba, SC, Brazil). A toothpaste solution was prepared by using the toothpaste Colgate Iluminous White (Colgate-Palmolive, São Bernardo do Campo, SP, Brazil) and distilled water at $1: 2$ ratios by weight. ${ }^{13,14}$ Specimens were undergone to 585 brushing cycles under a constant load of $200 \mathrm{~g}$, three times a day for 5 days. Each cycle was determined by the one back and forth movement of the brush. After the end of the abrasive challenge, the roughness and hardness of specimens were assessed again as described before.

\section{Qualitative Analysis of Surface}

Three specimens of each experimental condition were randomly analyzed by using a scanning electron microscope (Leica EM SCD50, Leica Microsystems, Wetzlar, Lahn-Dill, Germany). Specimens were sputter-coating with a thin film of gold at $15.0 \mathrm{Kv}$. The images were taken with $\times 1,000$ magnification selecting a more representative area in the specimen.

\section{Data Analysis}

Changes on roughness and hardness were calculated by subtracting the values measured after corrosive and/or abrasive challenges from those observed at baseline. Normal distribution of data and homogeneity of variance were assessed by Shapiro-Wilks and Levene's test, respectively. Data were analyzed by one-way ANOVA and post hoc Tukey's test. A confidence level of $95 \%$ was preset for all data analysis.

Table 1 Means (standard deviation) of changes on roughness and hardness of composite specimens according to time elapsed between the corrosive and abrasive challenges $(n=9)$

\begin{tabular}{|l|l|l|}
\hline Treatments & $\begin{array}{l}\text { Roughness } \\
\left(\mu \mathrm{m} \times 10^{-2}\right)\end{array}$ & $\begin{array}{l}\text { Hardness (knoop } \\
\text { microhardness) }\end{array}$ \\
\hline Control $^{\mathrm{a}}$ & $-9.4(10.9)^{\mathrm{A}}$ & $-6.1(14.3)$ \\
\hline No interval & $11.7(14.4)^{\mathrm{B}}$ & $-9.9(14.3)$ \\
\hline 15 minutes & $-3.74(11.8)^{\mathrm{A}, \mathrm{B}}$ & $-7.2(9.2)$ \\
\hline 30 minutes & $-5.81(11.7)^{\mathrm{A}}$ & $-10.3(13.0)$ \\
\hline
\end{tabular}

anly abrasive challenge.

Note: For roughness, distinct letters indicate statistical difference $(p<0.05)$. For hardness, there was no statistical differences among the treatments.

\section{Results}

The results for changes in roughness and hardness are summarized in - Table 1. One-way ANOVA showed that the treatment affected the changes on roughness of specimens $(p=0.005)$. The no interval specimens presented the highest values of change on roughness (11.7 [14.4]), but without statistical difference for elapsing 15 minutes (-3.74 [11.8]) between the challenges. There was no statistical difference between the intervals of 15 and 30 minutes ( -5.81 [11.7]), and the control (-9.4 [10.9]). One-way ANOVA showed that the treatment did not affect the changes on surface hardness $(p=0.858)$ for control (-6.1 [14.3]), no interval (-9.9 [14.3]), 15 minutes ( -7.2 [9.2]), and 30 minutes ( -10.3 [13.0]).

The smoothest surface was observed at baseline ( - Fig. 2A). The control group ( $\mathbf{- F i g}$. 2B) presented a smoother surface (no groove neither exposed fillers) than specimens underwent to corrosive challenge. No interval between the challenges (-Fig. 2C) yielded the deepest grooves and most pronounced irregularities on composite surface. Slight grooves and few irregularities were observed when an interval of 15 (-Fig. 2D) or 30 minutes (-Fig. 2E) were elapsed between the challenges.

\section{Discussion}

The acid beverage intake associated with brushing in sequence can generate changes on tooth surface ${ }^{10}$ and on surface of composite resin restoration, which was confirmed with present study. Therefore, the null hypothesis was rejected because the interval time between corrosive and abrasive challenges influence the surface roughness of nanofilled composite resins.

The worldwide consumption of soft drinks, fruit juice, sports drinks, and energy drinks has increased in recent decades. ${ }^{15}$ The consummation of some acidic beverages is associated with the healthy life style and to the improvement of the performance in physical exercises. ${ }^{16}$ The isotonic beverage is an acidic beverage widely used by athlete's aid the body maintain proper hydration and supplement minerals that are lost in sweat during excessive exercising. ${ }^{16}$ Despite these indications, the isotonic present a low pH (3.8) and it is associated with the capacity of dental wear and restorative materials. ${ }^{17}$ When the teeth are exposed to acid substances and with a low concentration of $\mathrm{Ca}^{2+}, \mathrm{PO}_{4}{ }^{3-}$, and $\mathrm{OH}^{-}$, there is a tendency for enamel to release more of these ions, and the demineralization process is more intense. ${ }^{15}$ During the oral intake, the acid beverage is swished in the mouth and result in higher contact of the beverage with the tooth and restorative surfaces. ${ }^{17}$ The agitation of the acid fluid in the oral cavity promotes the continuous ions outflow from the enamel and will lead to a more intensive corrosive-erosive process. $^{10,15}$

In addition, the lifestyles and the association with other factors such as parafunctional habits and occlusal factors 

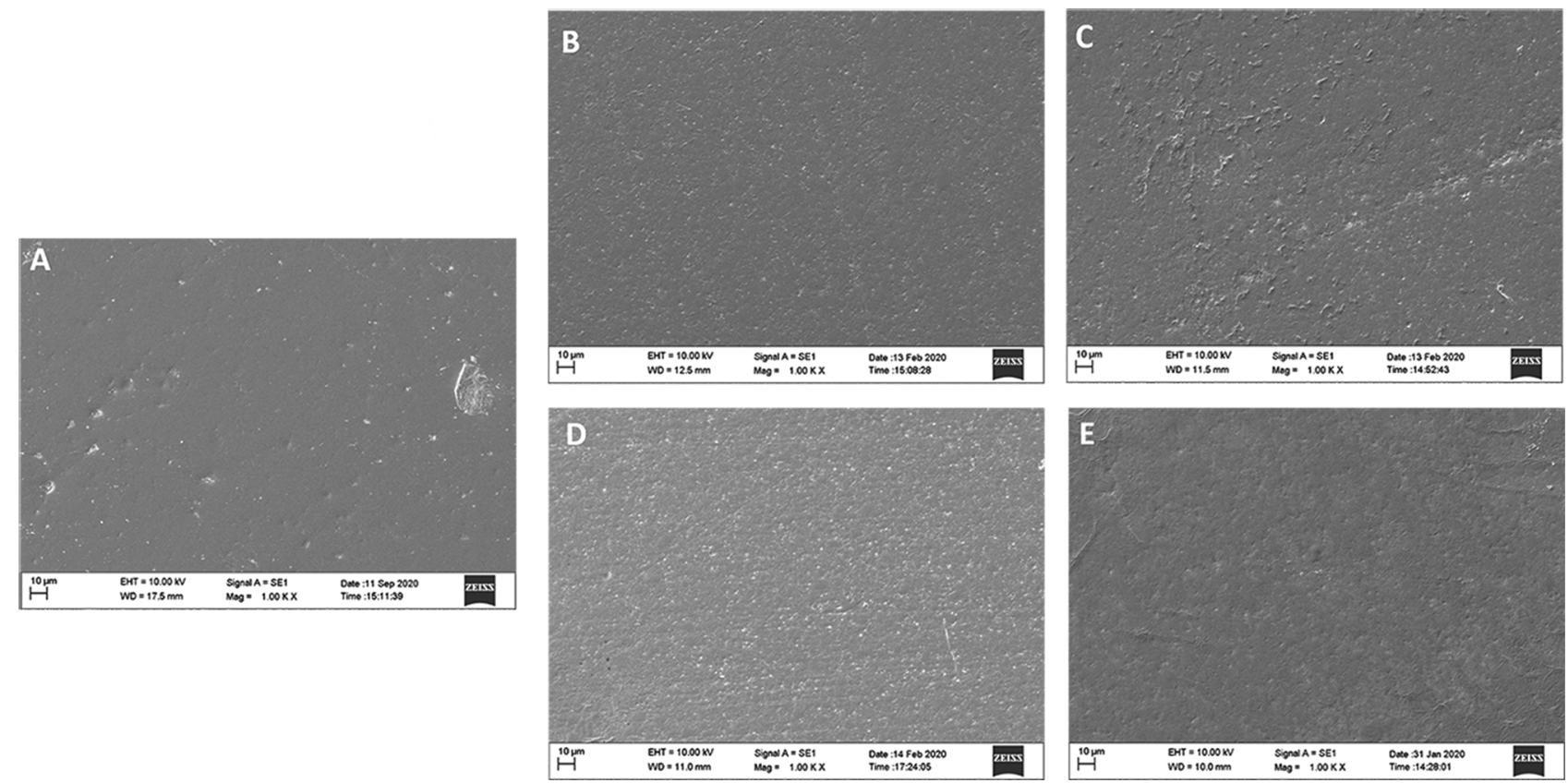

Fig. 2 Scanning electronic microscopies (x1000 magnification) illustrating the composiite surface before (A) and after corrosive and/or abrasive challenge; (B) only abrasive challenge (control); (C) no interval between the challenge; (D) interval of 15 minutes between the challenge; (E) interval of 30 minutes between the challenge.

generate dental wear at levels that require restorations to replace the loss of tooth structure. The most common approach for restoring severely worn teeth was the use of resin-based composite. ${ }^{18}$ The survival rate of composite resin restorations on worn teeth is $85 \%$ in 7 years. ${ }^{18}$ This restoration must survive in an environment that the teeth did not could resist, so it is essential that the patient changes their habits and perform all the precautions and care necessaries to maintain the quality of the restorations. ${ }^{9,12,18}$

The immediate group showed an increase in surface roughness after 5 days of challenges. The isotonic is an aggressive immersion media due to the presence of citric acid in its composition and generates a chemical degradation of the organic matrix with, consequently, increase of the surface roughness. ${ }^{12}$ Furthermore, the chemical degradation, induced by low $\mathrm{pH}$ solution, increases the damage promoted by tooth brushing. ${ }^{13}$ This was confirmed on the roughness surface values and SEM, which showed that brushing immediately after immersion in beverages with acidic $\mathrm{pH}$ increase the composite resin roughness. The immediate group showed higher values of roughness surface after the corrosive and abrasive challenges than others groups, confirming the greater degradation of the resin matrix.

The 30-minute group did not present statistically change in surface roughness than control. The immersion in artificial saliva after the corrosive challenge generated a neutralization of the acid environment, reducing the potential damage of the brushing and organic matrix degradation. ${ }^{11}$ In other hand, the 15-minute group present similar roughness with all groups. The similarity between the 15 minutes and the immediate group is due to the incomplete $\mathrm{pH}$ neutralization. However, the SEM images showed a reduced inorganic particle extruded in composite resin surface on 15 and
30 minutes, which explain the similarity between control on 15 and 30 minutes. The toothpastes are used for oral hygiene, but usually these toothpastes present secondary function such as whitening..$^{14}$ Many patients desire a smile with white teeth; however, instead they look for a professional bleaching, they choose for others alternatives as whiting toothpaste. The International Organization for Standardization recommends that the relative dentin abrasiveness (RDA) of a toothpaste does not exceed $250 .{ }^{19}$ The toothpaste abrasiveness is important to prevent extrinsic staining of teeth and composite resin restoration. ${ }^{7}$ Whitening toothpaste present higher RDA (175) than other toothpaste. ${ }^{19}$ Moreover, the toothpaste composition must be considered beyond the RDA because the ability to remove the extrinsic stains is also related to the chemical composition. ${ }^{20}$

The potential wear of composite resin is material dependent. Different composite resins are different influenced by abrasives pastes, ${ }^{21}$ although the filler size of the composite resin is not determinant in the degradation process. ${ }^{14}$ This result can be explained due to no difference among the whiting and conventional toothpaste. ${ }^{22}$ The nanofilled resin used in this study showed smoother surface after brushing with a whitening toothpastes compound with hydrated silica particles as abrasive. Moreover, the association of soft-bristle toothbrush and low-abrasive dentifrice did not increase roughness for nanofilled and microfilled materials. ${ }^{8}$

An inadequate oral environment, with episodes of corrosive and abrasive challenges, can promote some properties changes of the composite resin. ${ }^{22}$ The hardness of the composite resin is an important property for evaluating the resistance of this material to indentation. However, the interval time between the corrosive and abrasive challenges evaluated, not influence in hardness in this study. 
The microhardness is related to the composition and content of the particles. ${ }^{23}$

According to all these aspects addressed, the waiting time from acidic beverages intake until performing oral hygiene is important for maintaining the surface roughness of the restoration. Moreover, considering the limitations of this manuscript, future studies should be performed with a longer immersion time, different types of beverages, others composite resins and different toothpaste.

\section{Conclusion}

Therefore, with the limitations of this article, it is possible to conclude that the immersion in acidic beverage followed immediately brushing with whitening toothpastes, increased the surface roughness. The 30 minutes between intake of acidic beverages and brushing were important to decrease the deleterious effects of composite resin restauration. Microhardness not influenced by the interval between ingestion of acidic beverage and brushing.

\section{Funding}

This study was financed in part by the Coordination for the Improvement of Higher Education Personnel (CAPES), Brazil, Finance Code 001

Conflict of Interest

None declared.

\section{References}

1 Demarco FF, Collares K, Correa MB, Cenci MS, Moraes RR, Opdam NJ. Should my composite restorations last forever? Why are they failing? Braz Oral Res 2017;31(Suppl 1):e56

2 Ferracane JL. Resin composite-state of the art. Dent Mater 2011;27(1):29-38

3 Da Rosa Rodolpho PA, Donassollo TA, Cenci MS, et al. 22-Year clinical evaluation of the performance of two posterior composites with different filler characteristics. Dent Mater 2011;27(10):955-963

4 Hickel R, Peschke A, Tyas M, et al. FDI World Dental Federation: clinical criteria for the evaluation of direct and indirect restorations-update and clinical examples. Clin Oral Investig 2010;14(4):349-366

5 Schroeder T, da Silva PB, Basso GR, Franco MC, Maske TT, Cenci MS. Factors affecting the color stability and staining of esthetic restorations. Odontology 2019;107(4):507-512

6 Mei L, Busscher HJ, van der Mei HC, Ren Y. Influence of surface roughness on streptococcal adhesion forces to composite resins. Dent Mater 2011;27(8):770-778

7 Zhao X, Zanetti F, Wang L, et al. Effects of different discoloration challenges and whitening treatments on dental hard tissues and composite resin restorations. J Dent 2019;89: 103182
8 Mozzaquatro LR, Rodrigues CS, Kaizer MR, Lago M, Mallmann A, Jacques LB. The effect of brushing and aging on the staining and smoothness of resin composites. J Esthet Restor Dent 2017;29(2):E44-E55

9 Erdemir U, Yildiz E, Eren MM, Ozel S. Surface hardness of different restorative materials after long-term immersion in sports and energy drinks. Dent Mater J 2012;31(5):729-736

10 Grippo JO, Simring M, Coleman TA. Abfraction, abrasion, biocorrosion, and the enigma of noncarious cervical lesions: a 20-year perspective. J Esthet Restor Dent 2012;24(1):10-23

11 Guedes AP, Oliveira-Reis B, Catelan A, Suzuki TYU, Briso ALF, Santos PHD. Mechanical and surface properties analysis of restorative materials submitted to erosive challenges in situ. Eur J Dent 2018;12(4):559-565

12 Pinelli MD, Catelan A, de Resende LF, Soares LE, Aguiar FH, Liporoni PC. Chemical composition and roughness of enamel and composite after bleaching, acidic beverages and toothbrushing. J Clin Exp Dent 2019;11(12):e1175-e1180

13 Lemos CA, Mauro SJ, Dos Santos PH, Briso AL, Fagundes TC. Influence of mechanical and chemical degradation in the surface roughness, gloss, and color of microhybrid composites. J Contemp Dent Pract 2017;18(4):283-288

14 de Moraes Rego Roselino L, Tonani Torrieri R, Sbardelotto C, et al. Color stability and surface roughness of composite resins submitted to brushing with bleaching toothpastes: an in situ study. J Esthet Restor Dent 2019;31(5):486-492

15 Saads Carvalho T, Lussi A. Chapter 9: acidic beverages and foods associated with dental erosion and erosive tooth wear. Monogr Oral Sci 2020;28:91-98

16 Broughton D, Fairchild RM, Morgan MZ. A survey of sports drinks consumption among adolescents. $\mathrm{Br}$ Dent J 2016;220(12):639-643

17 Ostrowska A, Szymański W, Kołodziejczyk Ł, BołtaczRzepkowska E. Evaluation of the erosive potential of selected isotonic drinks: in vitro studies. Adv Clin Exp Med 2016;25(6):1313-1319

18 Al-Khayatt AS, Ray-Chaudhuri A, Poyser NJ, et al. Direct composite restorations for the worn mandibular anterior dentition: a 7-year follow-up of a prospective randomised controlled split-mouth clinical trial. J Oral Rehabil 2013;40(5):389-401

19 Silva EMD, Maia JNDSMD, Mitraud CG, Russo JDES, Poskus LT, Guimarães JGA. Can whitening toothpastes maintain the optical stability of enamel over time? J Appl Oral Sci 2018;26:e20160460

20 Amaral CM, Rodrigues JA, Erhardt MC, et al. Effect of whitening dentifrices on the superficial roughness of esthetic restorative materials. J Esthet Restor Dent 2006;18(2):102-108, discussion 109

21 Liebermann A, Spintzyk S, Reymus M, Schweizer E, Stawarczyk B. Nine prophylactic polishing pastes: impact on discoloration, gloss, and surface properties of a CAD/CAM resin composite. Clin Oral Investig 2019;23(1):327-335

22 Roque AC, Bohner LO, de Godoi AP, Colucci V, Corona SA, Catirse AB. Surface roughness of composite resins subjected to hydrochloric acid. Braz Dent J 2015;26(3):268-271

23 Mara da Silva T, Barbosa Dantas DCB, Franco TT, Franco LT, Rocha Lima Huhtala MF. Surface degradation of composite resins under staining and brushing challenges. J Dent Sci 2019;14(1):87-92 